\title{
Langer's axillary arch: a rare variant, and prevalence among Caucasians
}

\author{
M.A. Turki, P.J. Adds \\ Institute of Medical and Biomedical Education (Anatomy), St. George's, University of London, United Kingdom \\ [Received: 27 October 2016; Accepted: 29 November 2016]
}

\begin{abstract}
During the dissection of a 79-year-old Caucasian female cadaver, a variant of Langer's axillary arch was found unilaterally in the left axilla. While Langer's axillary arches are not uncommon, this particular variant, attaching to the biceps brachii, is much rarer with a reported prevalence of only $0.25 \%$. The case reported here, however, is only the third example of a Langer's axillary arch that has been found in the last 14 years in the Dissecting Room at St. George's, University of London, giving it an overall prevalence of approximately $1.0 \%$ amongst a population of around 280 Caucasian cadavers, much lower than the reported frequency of 7\%. Langer's axillary arches can be completely asymptomatic in life, but may also cause a variety of issues both clinically and surgically. (Folia Morphol 2017; 76, 3: 536-539)
\end{abstract}

Key words: axillary arch variant, oncoplastic surgery, lymphadenectomy

\section{INTRODUCTION}

Over the past 200 years, anatomical variations of the axillary region have been reported not only in textbooks of human anatomy but also in those of operative surgery. The surgical importance of the axillary region in many procedures, including breast reconstruction, lymphadenectomies and oncoplastic surgery has led to a rise in interest in axillary variations.

One of the muscular variants in the axillary region is a muscle slip running from the lateral border of latissimus dorsi across the axillary neurovascular bundle to the pectoralis major, which has variously been termed the pectodorsal muscle, arcus axillaris, and Langer's axillary arch $[1,5]$.

Nowadays, the term "Langer's axillary arch", first coined by Testut in 1884, describes any muscular anatomical variant running from the lateral border of latissimus dorsi to various points anterolateral to the humerus. Historically, the axillary arch was first described in 1783 by Bugnone, then again by Ramsay in 1793 and then finally by Langer in 1846 [see 3]. It should be noted, however, that both arches mentioned by Bugnone and Ramsay [see 4] were muscular in nature, while Langer specifically mentions a fibrous variant, suggesting that Langer's axillary arch can have various degrees of muscularisation and is not simply either muscle or fibrous.

The presence of an axillary arch can lead to confusion in physical examination and imaging, and complications during surgery to the axilla, such as breast reconstruction, bypass surgery or lymph node dissection, and is something of which all surgeons operating in the region should be aware.

\section{CASE REPORT}

A dissection was performed on the axillae of a formalin-fixed, 79-year-old, Caucasian female cadaver. The cause of death was listed as metastatic lung cancer (unrelated to the arch). The body had been donated to the Anatomy Department of St. George's,

Address for correspondence: Dr. P.J. Adds, IMBE (Anatomy), St. George's University of London, Cranmer Terrace, London SW170RE, United Kingdom, tel: +44 (0)208 7255208, e-mail: padds@sgul.ac.uk 


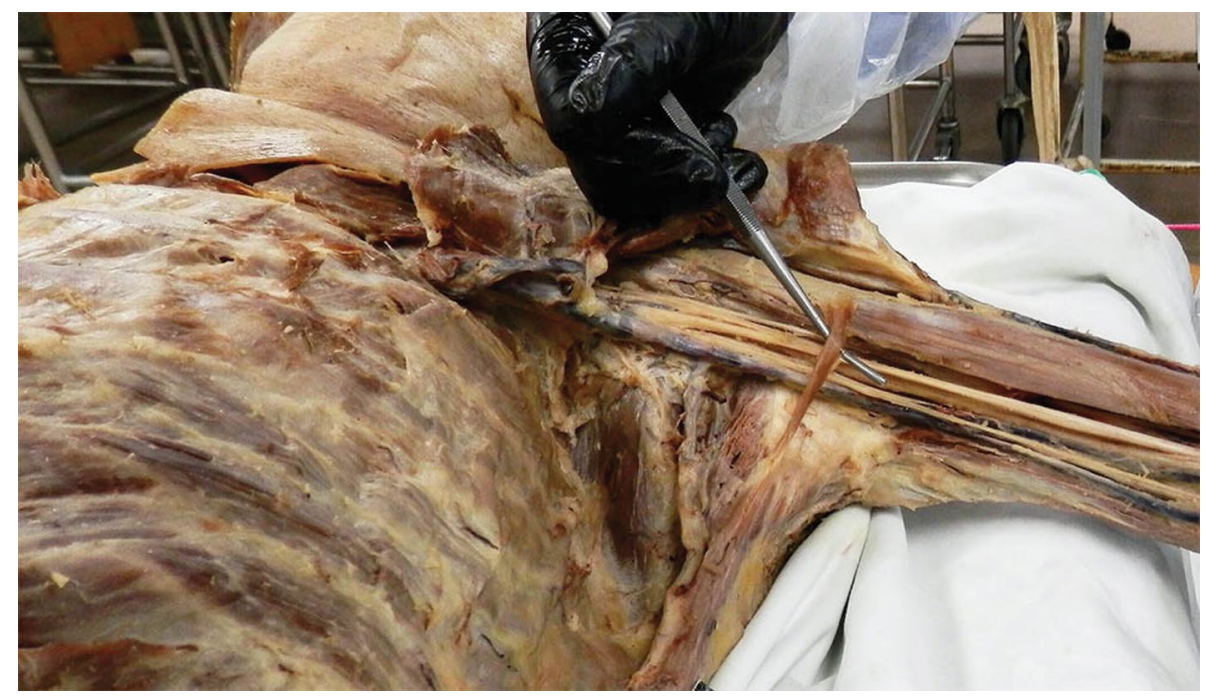

Figure 1. The axillary arch, inferior view. Pectoralis major and minor have been reflected. Probe indicates axillary arch.

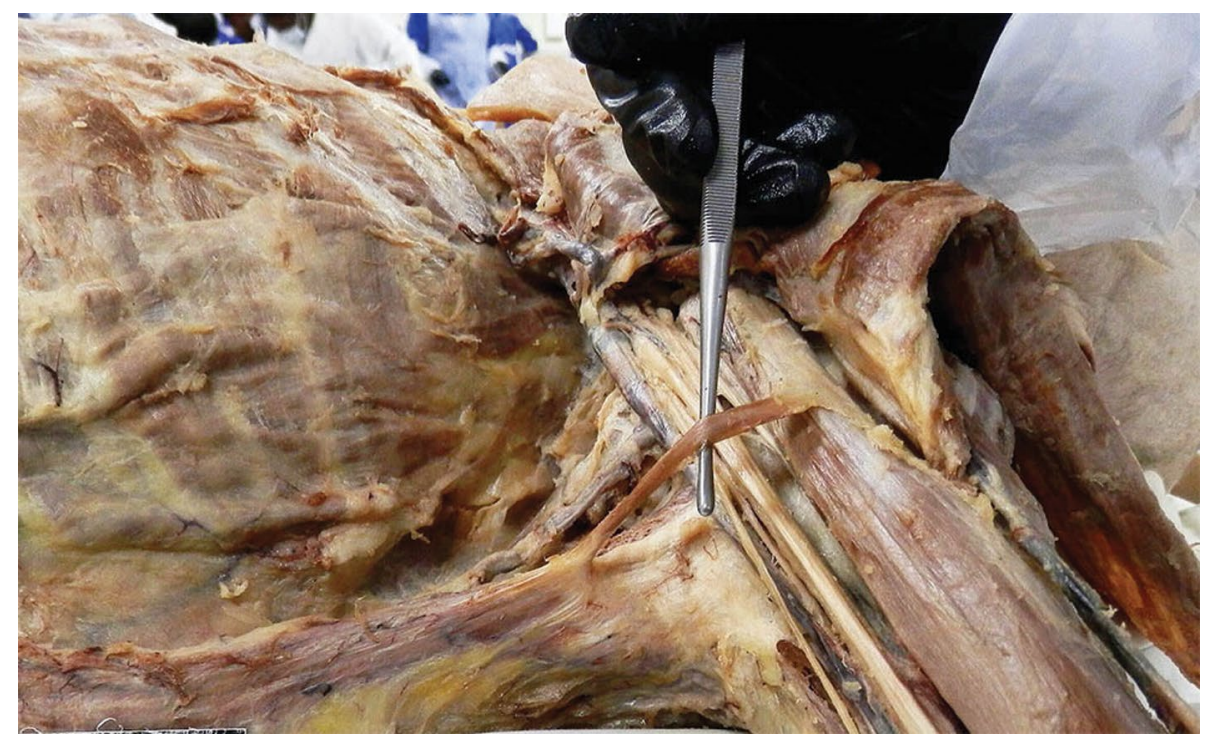

Figure 2. Left axilla, lateral view. Pectoralis major and minor have been reflected. The probe indicates the arch crossing the neurovascular bundle.

University of London under the Human Tissue Act (2004). The dissection revealed a left-sided unilateral Langer's axillary arch (Fig. 1).

The left axilla was dissected and cleared of fat to reveal the brachial plexus, axillary vessels, and muscles of the upper limb. A slip of muscle was revealed, running from the anterior border of latissimus dorsi across the neurovascular bundle in the axilla to a fibrous attachment on the proximal part of the short head of the biceps brachii (Figs. 1, 2)

\section{DISCUSSION}

Axillary arches are important anatomical variants that have a reported frequency of about 7\% [9]. However, its frequency varies significantly between populations: $1.7 \%$ among Turkish individuals, in contrast to $43.8 \%$ in a Chinese population [3]. This suggests that its prevalence is highly variable, and directly correlated to ethnicity. The unusual variant described here has a reported prevalence of $0.25 \%$ in the Caucasian population [8]. However, 
this was the only the third example of a Langer's axillary arch seen in around 280 Caucasian cadavers in St. George's, University of London, giving it an overall prevalence of approximately $1.0 \%$, significantly lower than the reported prevalence of $7 \%$. The variant described here has been seen once in the same number of cadavers, giving this particular variant a prevalence of approximately $0.36 \%$ among Caucasians.

Variations of the arch have been reported, inserting into different areas, such as the anterior surface of pectoralis major, pectoralis minor, the axillary fascia, the fascia of coracobrachialis, the fascia covering the biceps brachii, the long head of the biceps brachii muscle, the coracoid process and to the greater tubercle of the humerus $[3,5]$.

The three main features that characterise Langer's axillary arches as we understand them today are as follows: they originate from the latissimus dorsi, insert at the anterosuperior region of the humerus, and cross the neurovascular bundle of the axilla from dorsomedial to ventrolateral [3].

The arch carries various surgical implications of differing severity and urgency. Firstly, the arch could be the cause of confusion during lymphadenectomy as it could be mistaken for a tumorous lymph node. The arch could also hinder the progress of bypass surgery due to the restriction of access it causes in the axilla region. Furthermore, during lymph node dissection, the most common procedure involving the axilla in breast cancer patients, there could be an incomplete clearance because of the restricted exposure of the lower lateral group of lymph nodes. A more extreme surgical consequence of the axillary arch is ischaemic necrosis induced by the stretching or compressing effect of the arch on the thoracodorsal pedicle during a latissimus dorsi musculocutaneous flap breast reconstruction surgery [4].

The arch could also present a range of clinical implications of differing magnitude and severity. Firstly, it can make the distinction and palpation of axillary lymph nodes difficult for physicians during clinical examinations. It can also contribute to hyperabduction syndrome where the patient experiences numbness and/or pain in the fingers due entrapment of nerves in the brachial plexus. It could also lead to costoclavicular syndrome in which pain and stiffness in the neck and shoulder are experienced, accompanied by puffy blue hands, due to the entrapment of the axillary vein by the arch. There have also been reported instances where the involvement of an axillary arch has been shown to catalyse the development of deep vein thromboses [6]. A more serious and debilitating outcome is the inability of some patients to move their arm in the cases where the axillary arch develops a contracture. The cure to all these symptomatic cases is a simple surgical transection of the muscular slip [3].

Although the arch may be observed as a swelling during examination of the axillary region in some patients [7], imaging techniques can also be used to confirm its existence prior to surgical procedures involving the axilla. The arch, however, can also be a hindrance in imaging procedures where it overshadows lymph nodes and other important structures [3]. Radiologists' increased familiarity with the arch can minimise the number of potential complications by improving preoperative detection rates so that the surgical team can be made aware in advance, allowing them to make adjustments and accommodate their approach accordingly [2].

\section{CONCLUSIONS}

In conclusion, this case report and literature review discusses an unusual presentation of Langer's axillary arch, a clinically relevant anatomical variant, and gives a new estimate of the prevalence of the arch in the Caucasian population. If unnoticed or misidentified, Langer's axillary arch can lead to inaccurate diagnoses and surgical complications, and reinforces why thorough anatomical knowledge of the axillary region and its variations, and appropriate surgical skills are vital in avoiding clinical and surgical complications, as well as correctly identifying symptomatic conditions.

\section{Acknowledgements}

The authors would like to acknowledge the generosity of the donor of this cadaver without whom this study would not have been possible.

\section{REFERENCES}

1. Besana-Ciani I, Greenall MJ. Langer's axillary arch: anatomy, embryological features and surgical implications. Surgeon. 2005; 3(5): 325-327, doi: 10.1016/s1479666x(05)80111-, indexed in Pubmed: 16245651.

2. Guy MS, Sandhu SK, Gowdy JM, et al. MRI of the axillary arch muscle: prevalence, anatomic relations, and potential consequences. AJR Am J Roentgenol. 2011; 196(1): W52-W57, doi: 10.2214/AJR.10.4380, indexed in Pubmed: 21178031. 
3. Hirtler L. Langer's axillary arch - case presentation and literature overview. Austin J Anat. 2014; 1: 1-5.

4. Jelev L, Georgiev GP, Surchev L. Axillary arch in human: common morphology and variety. Definition of "clinical" axillary arch and its classification. Ann Anat. 2007; 189(5): 473-481, doi: 10.1016/j.aanat.2006.11.011, indexed in Pubmed: 17910401.

5. Kalaycioglu A, Gümüsalan Y, Ozan H. Anomalous insertional slip of latissimus dorsi muscle: arcus axillaris. Surg Radiol Anat. 1998; 20(1): 73-75, doi: 10.1007/ bf01628121, indexed in Pubmed: 9574494.

6. Magee C, Jones C, McIntosh S, et al. Upper limb deep vein thrombosis due to Langer's axillary arch. J Vasc Surg. 2012;
55(1): 234-236, doi: 10.1016/j.jvs.2011.07.002, indexed in Pubmed: 21924577.

7. Saitta GF, Baum V. Langer's axillary arch. An unusual cause of axillary mass. JAMA. 1962; 180: 690, doi: 10.1001/jama.1962.03050210052017, indexed in Pubmed: 14496135.

8. Serpell JW, Baum M. Significance of 'Langer's axillary arch' in axillary dissection. Aust N Z J Surg. 1991; 61(4): 310-312, doi: 10.1111/j.1445-2197.1991.tb00218x, indexed in Pubmed: 2018442.

9. Warwick R, Williams PL. (eds.) Gray's Anatomy. 35th Ed. Longman, Edinburgh 1973: 534. 